\title{
Urinary tract infection is associated with hypokalemia: a case control study
}

\author{
Ai-Ling Shen ${ }^{1}$, Hsiu-Li Lin ${ }^{1,2}$, Hsiu-Chen Lin ${ }^{3,4}$, Yuan-Fu Tseng ${ }^{1}$, Chien-Yeh Hsü ${ }^{2,5}$ and Che-Yi Chou ${ }^{6,7,8^{*}}$ (D)
}

\begin{abstract}
Background: Hypokalemia is a common clinical problem. The association between urinary tract infection (UTI) and hypokalemia is not clear. Hypokalemia is common in patients with UTI in clinical observation. The aim of the study is to determine if UTI is associated with hypokalemia.

Methods: Patients hospitalized with UTI and the control group were retrieved from the Longitudinal Health Insurance Database 2005. The control group was patients hospitalized with other reasons and were matched for the confoundings of UTI and hypokalemia. We analyze the risk of hypokalemia using logistic regression and calculate the odds ratio (OR) and 95\% confidence interval (CI) of OR.
\end{abstract}

Results: We analyzed 43,719 UTI patients and control patients. Hypokalemia was found in 4540 (10.4\%) patients with UTI and $1842(4.2 \%)$ control patients. The percentage of patients with hypokalemia was higher in UTI patients (chi-square, $p<0.001$ ). UTI was associated with hypokalemia and the odds ratio (OR) was 2.27 [95\% confidence interval (Cl): 2.17-2.41]. Cerebrovascular accident, chronic obstructive pulmonary disease, hypertension, congestive heart failure, diarrhea, medications including thiazides, sulfonamides, xanthines, and laxatives were independently associated with hypokalemia. Recurrent UTI was associated with hypokalemia in UTI patients (OR: 1.13, 95\% Cl: 1.05$1.23, p<0.001)$.

Conclusions: Urinary tract infection is associated with hypokalemia among inpatients. The association is independent of patients' comorbidities and medications. Recurrent UTI is associated with increased hypokalemia in UTI patients.

Keywords: UTI, Hypokalemia, Recurrent UTI, Comorbidities, Diarrhea, Thiazides, Sulfonamides

\section{Background}

Hypokalemia is a common electrolyte abnormality in hospitalized patients. Unrecognized hypokalemia may trigger arrhythmias [1] and is associated with increased mortality in patients with chronic diseases such as heart failure, diabetes, or chronic kidney disease [2,3]. Urinary tract infection (UTI) is a common bacterial infection in women and the elderly [4-6]. Clinical manifestations of

\footnotetext{
* Correspondence: cychou.chou@gmail.com

${ }^{6}$ Division of Nephrology, Asia University Hospital, NO 222, Fuxin Rd, Wufeng Dist, Taichung 413, Taiwan

${ }^{7}$ Department of Post-baccalaureate Veterinary Medicine, Asia University, NO

222, Fuxin Rd, Wufeng Dist, Taichung 413, Taiwan

Full list of author information is available at the end of the article
}

UTI include dysuria, frequency, urgency, fever, nausea, vomit, and gross hematuria. It may not be surprised that hypokalemia may be occurred because of increased potassium loss after vomit and fever. However, the association between UTI and hypokalemia is not clear [7-11]. We observed that hypokalemia is common among UTI patients in clinical settings. This study was conducted to determine the association between UTI and hypokalemia. Inpatients were selected because serum potassium is rarely measured in outpatient settings as UTI has not been considered as a risk factor for hypokalemia.

(c) The Author(s). 2020 Open Access This article is licensed under a Creative Commons Attribution 4.0 International License, which permits use, sharing, adaptation, distribution and reproduction in any medium or format, as long as you give appropriate credit to the original author(s) and the source, provide a link to the Creative Commons licence, and indicate if changes were made. The images or other third party material in this article are included in the article's Creative Commons licence, unless indicated otherwise in a credit line to the material. If material is not included in the article's Creative Commons licence and your intended use is not permitted by statutory regulation or exceeds the permitted use, you will need to obtain permission directly from the copyright holder. To view a copy of this licence, visit http://creativecommons.org/licenses/by/4.0/ The Creative Commons Public Domain Dedication waiver (http://creativecommons.org/publicdomain/zero/1.0/) applies to the data made available in this article, unless otherwise stated in a credit line to the data. 


\section{Methods}

We extracted data from the Longitudinal Health Insurance Database 2005 (LHID2005) as prescribed in the previous study [12]. The dataset used in this study is a de-identified secondary data and is released to the public for research purposes. A review of the institutional review board is not required. All inpatients with UTI between 1 January 2000 and 31 December 2008 were extracted. The first hospitalization for UTI was selected in the patients with more than one episode of UTI. The control patients were patients who are hospitalized for other reasons with matching for confoundings at a 1:1 ratio. The confoundings include cerebrovascular accident (CVA), chronic obstructive pulmonary disease (COPD), diabetes mellitus (DM), hypertension (HTN), acute kidney injury (AKI), chronic kidney disease (CKD), Cushing syndrome, hyperthyroidism, leukorrhea, medications, and urethral catheterization. These confoundings were used because they are associated with UTI or hypokalemia in the literature.

The patients hospitalized with UTI were identified with ICD-9-CM code 599 on discharge. Patients on dialysis were excluded in this study because pyuria is common in auric dialysis patients. The development of hypokalemia was identified using ICD-9-CM code (276.8) in the same hospitalization. The comorbidities diagnosed before the episode of UTI in at least 3 visits were retrieved. The comorbidities include CVA (ICD-9CM code 430-436 and 438), COPD (490-496), DM (250), HTN (401-405), CHF (428), AKI (584.9), CKD (585), Cushing's syndrome (255.0), hyperthyroidism (242), hypothyroidism (244), alkalosis (276.3), diarrhea (787.91), malnutrition $(269.9,262,263)$, poor intake (783.0), magnesium disorder (275.2), and leukorrhea (616 and 623.5). Urethral catheterization in 1 week before hospitalization was identify using procedure code 47013-47014. Recurrent UTI was defined as individuals who had more than two episodes of UTI in 6 months or three episodes in 1 year [13]. Medications used for more than 14 days in 1 month before the UTI diagnosed were extracted. Medications include potassium-sparing diuretics (ATC code C03D, C03E), thiazides (C03A, C03B), sulfonamides (C03CA01, C03CA02), beta-2adrenoreceptor agonists (R03CC), xanthines (R03DA, R03DB), antibiotics (J01CA, J01CR, J01GB, A01AB04), meropenem (J01DH02), insulin (A10), steroids (H02AB), potassium binders (V03AE01), and laxatives (A06AG07).

\section{Statistical analysis}

Data are expressed as number (percentage), or mean (standard deviation) where appropriate. Chi-square tests and $t$-test was used to test the differences of variables between two groups. The association of hypokalemia and UTI was estimated using univariable logistic regression. Variables with a $p<0.05$ in univariable logistic regression were further analyzed by multivariable logistic regression. The odds ratios (ORs) and 95\% confidence interval (CIs) of ORs were calculated. All statistical analyses were carried out using SAS statistical package, version 9.3 (SAS, Inc., NC, USA). All comparison tests were two-sided and a $P$-value of less than 0.05 was considered as statistically significant.

\section{Results}

A total of 43,719 inpatients with UTI and matched control patients were analyzed (Table 1). 4540 (10.4\%) UTI patients and $1842(4.2 \%)$ control patients had hypokalemia $(p<0.001$, chi-square test). The duration of hospitalization was longer in UTI patients $12.7 \pm 24.8$ days vs $7.4 \pm 14.5$ days in the control patients $(p<0.001)$. The average age of UTI patients was $63.5 \pm 17.7$ years old and $63.6 \pm 18.2$ years old in the control patients $(p=$ 0.492). $39.3 \%$ of UTI patients and $39.2 \%$ of control patients were male. The percentage of patients with different comorbidities were similar in UTI patients and control patients after matching. The percentage of CVA was $28.8 \%$ in UTI patients and $28.4 \%$ in control patients. COPD 30.9 and 30.7\%, DM 33.8 and 33.4\%, HTN 59.2 and 58.9\%, CHF 14.1 and $14.0 \%$, AKI 0.3 and $0.3 \%$, CKD 7.2 and $7.2 \%$. Diarrhea, poor intake, and malnutrition were identified in $0.1 \%$ of the patients. The percentage of patients on potassium-sparing diuretics was $0.1 \%$ in UTI patients and $0.2 \%$ in control patients. Thiazides 0.6 and $0.6 \%$, sulfonamides 0.4 and $0.5 \%$, beta-2adrenoreceptor agonists 0.7 and $0.7 \%$, xanthiums 4.1 and $4.1 \%$, antibiotics 0.7 and $0.7 \%$, insulin 1.7 and $1.7 \%$, steroids 1.5 and $1.5 \%$, potassium binders 0.2 and $0.2 \%$, laxatives 10.2 and $10.1 \%$. The percentage of patients with urethral catheterization was $6.7 \%$ in both groups.

UTI was associated with hypokalemia in univariable and multivariable logistic regression (Table 2). The ORs were 2.48 (95\% CI: $2.33-2.64, p<0.001$ ) in univariable logistic regression and 2.27 (95\% CI: 2.13-2.41, $p<0.001$ ) in multivariable logistic regression. Factors associated with hypokalemia in univariable logistic regression include age, CVA, COPD, DM, HTN, CHF, CKD, diarrhea, potassiumsparing diuretics, thiazides, sulfonamides, beta-2adrenoreceptor agonists, xanthines, steroids, and laxatives. DM, CKD, beta-2-adrenoreceptor agonists, and steroids were not significantly associated with hypokalemia in multivariable logistic regression. The ORs of age was 1.01 (95\% CI: 1.01-1.01) for every one additional year, CVA 1.29 (95\% CI: 1.20-1.39), COPD 1.47 (95\% CI: 1.37-1.57), HTN 1.56 (95\% CI: 1.44-1.68), CHF 1.40 (95\% CI: 1.281.53), diarrhea 2.25 (95\% CI: 1.24-3.82), thiazides 1.57 (95\% CI: 1.21-2.00), sulfonamides 1.44 (95\% CI: $1.03-$ 1.97), xanthines 1.44 (95\% CI: 1.22-1.69), and laxatives 1.32 (95\% CI: 1.16-1.52). 
Table 1 Clinical characteristics of patients with and without urinary tract infection (UTI)

\begin{tabular}{|c|c|c|c|}
\hline Factor & UTI $N=43,719$ & Control $N=43,719$ & $P$ \\
\hline Hypokalemia n (\%) & $4540(10.4)$ & $1842(4.2)$ & $<0.001$ \\
\hline Duration of hospitalization (day) & $12.7 \pm 24.8$ & $7.4 \pm 14.5$ & $<0.001$ \\
\hline Age & $63.6 \pm 18.2$ & $63.5 \pm 17.7$ & 0.492 \\
\hline Male n (\%) & $17,177(39.3)$ & $17,152(39.2)$ & 0.87 \\
\hline \multicolumn{4}{|l|}{ Comorbidity n (\%) } \\
\hline CVA & $12,574(28.8)$ & $12,412(28.4)$ & 0.23 \\
\hline COPD & $13,519(30.9)$ & $13,428(30.7)$ & 0.51 \\
\hline DM & $14,776(33.8)$ & $14,615(33.4)$ & 0.25 \\
\hline HTN & $25,860(59.2)$ & $25,752(58.9)$ & 0.46 \\
\hline $\mathrm{CHF}$ & $6154(14.1)$ & $6128(14.0)$ & 0.81 \\
\hline AKI & $119(0.3)$ & $117(0.3)$ & 0.95 \\
\hline CKD & $3162(7.2)$ & $3125(7.1)$ & 0.64 \\
\hline Cushing syndrome & $283(0.6)$ & $279(0.6)$ & 0.90 \\
\hline Hyperthyroidism & $660(1.5)$ & $654(1.5)$ & 0.89 \\
\hline Leukorrhea & $350(0.8)$ & $332(0.8)$ & 0.51 \\
\hline Diarrhea & $59(0.1)$ & $62(0.1)$ & 0.85 \\
\hline Poor intake & $40(0.1)$ & $46(0.1)$ & 0.59 \\
\hline Malnutrition & $22(0.05)$ & $25(0.06)$ & 0.77 \\
\hline \multicolumn{4}{|l|}{ Medications n (\%) } \\
\hline Potassium-sparing diuretics & $63(0.1)$ & $75(0.2)$ & 0.35 \\
\hline Thiazides & $261(0.6)$ & $283(0.6)$ & 0.37 \\
\hline Sulfonamides & $175(0.4)$ & $208(0.5)$ & 0.10 \\
\hline Beta-2-adrenoreceptor agonists & $176(0.4)$ & $204(0.5)$ & 0.17 \\
\hline Xanthiums & $1777(4.1)$ & $1782(4.1)$ & 0.94 \\
\hline Antibiotics & $298(0.7)$ & $296(0.7)$ & 0.97 \\
\hline Insulin & $752(1.7)$ & $763(1.7)$ & 0.79 \\
\hline Steroids & $648(1.5)$ & $663(1.5)$ & 0.69 \\
\hline Potassium binders & $105(0.2)$ & $105(0.2)$ & - \\
\hline Laxatives & $4458(10.2)$ & $4412(10.1)$ & 0.61 \\
\hline Urethral catheterization & $3034(6.7)$ & $3034(6.7)$ & - \\
\hline
\end{tabular}

CVA cerebrovascular accident, COPD chronic obstructive pulmonary disease, DM diabetes mellitus, HTN hypertension, AKI acute kidney injury, CKD chronic kidney disease, $\mathrm{CHF}$ congestive heart failure

Recurrent UTI was significantly associated with hypokalemia in UTI patients (Table 3). The ORs were 1.31 (95\% CI: $1.21-1.41)$ in univariable logistic regression and 1.13 (95\% CI: 1.05-1.23) in multivariable logistic regression. Patient's age, CVA, COPD, HTN, CHF, thiazides, sulfonamides, xanthines, and laxatives were independently associated with hypokalemia in UTI patients in univariable logistic regression, but diarrhea was not. The OR of age in the multivariable logistic regression was 1.01 (95\% CI: 1.01-1.01), CVA 1.16 (95\% CI: $1.08-1.24$ ), COPD 1.45 (95\% CI: $1.35-$ 1.55), HTN 1.42 (95\% CI: 1.31-1.53), CHF 1.41 (95\% CI: 1.31-1.53), xanthines 1.42 (95\% CI: 1.25-1.62), and laxatives 1.29 (95\% CI: 1.17-1.41).

\section{Discussion}

In this case-control cross-sectional study, we demonstrated the association between UTI and hypokalemia among inpatients. This finding is supported by the increased OR of hypokalemia in the multivariable logistic regression. The association between hypokalemia and UTI is independent of comorbidities and mediations. Besides, recurrent UTI is also associated with hypokalemia in UTI patients. Hypokalemia in UTI may explain the cardiac arrest following infectious disease in the previous study [14]. Inpatients with UTI were selected in this study because the serum potassium was more likely to be measured in inpatient settings. The development of hypokalemia in the patients with UTI may be explained 
Table 2 Factors associated with hypokalemia in univariable and multivariable logistic regression

\begin{tabular}{|c|c|c|c|c|c|c|}
\hline \multirow[t]{2}{*}{ Factors } & \multicolumn{3}{|c|}{ Univariable } & \multicolumn{3}{|c|}{ Multivariable } \\
\hline & ORs & $95 \%$ & $\mathrm{Cls}$ & ORs & $95 \%$ & $\mathrm{Cls}$ \\
\hline$\overline{\text { UTI }}$ & 2.48 & 2.33 & 2.64 & 2.27 & 2.13 & 2.41 \\
\hline Age & 1.03 & 1.03 & 1.03 & 1.01 & 1.01 & 1.01 \\
\hline \multicolumn{7}{|l|}{ Comorbidity } \\
\hline CVA & 2.29 & 2.14 & 2.44 & 1.29 & 1.20 & 1.39 \\
\hline COPD & 2.21 & 2.08 & 2.34 & 1.47 & 1.37 & 1.57 \\
\hline $\mathrm{DM}$ & 1.56 & 1.46 & 1.66 & 1.02 & 0.95 & 1.09 \\
\hline HTN & 2.62 & 2.46 & 2.79 & 1.56 & 1.44 & 1.68 \\
\hline $\mathrm{CHF}$ & 2.49 & 2.28 & 2.71 & 1.40 & 1.28 & 1.53 \\
\hline CKD & 1.42 & 1.21 & 1.66 & 0.98 & 0.84 & 1.15 \\
\hline Diarrhea & 2.29 & 1.28 & 3.82 & 2.25 & 1.24 & 3.82 \\
\hline \multicolumn{7}{|l|}{ Medications } \\
\hline Potassium-sparing diuretics & 1.96 & 1.12 & 3.19 & 1.24 & 0.69 & 2.08 \\
\hline Thiazides & 1.98 & 1.53 & 2.51 & 1.57 & 1.21 & 2.00 \\
\hline Sulfonamides & 2.26 & 1.64 & 3.06 & 1.44 & 1.03 & 1.97 \\
\hline Beta-2-adrenoreceptor agonists & 2.04 & 1.48 & 2.76 & 0.91 & 0.64 & 1.26 \\
\hline Steroids & 1.57 & 1.29 & 1.89 & 1.12 & 0.91 & 1.37 \\
\hline Xanthines & 2.38 & 2.05 & 2.74 & 1.44 & 1.22 & 1.69 \\
\hline Laxatives & 2.07 & 1.81 & 2.35 & 1.32 & 1.16 & 1.52 \\
\hline
\end{tabular}

Table 3 Risk of hypokalemia among patients with urinary tract infection

\begin{tabular}{|c|c|c|c|c|c|c|}
\hline \multirow[t]{2}{*}{ Factors } & \multicolumn{3}{|c|}{ Univariable } & \multicolumn{3}{|c|}{ Multivariable } \\
\hline & ORs & $95 \%$ & $\mathrm{Cls}$ & ORs & $95 \%$ & $\mathrm{Cls}$ \\
\hline Recurrent UTI & 1.31 & 1.21 & 1.41 & 1.13 & 1.05 & 1.23 \\
\hline Age & 1.02 & 1.02 & 1.03 & 1.01 & 1.01 & 1.01 \\
\hline \multicolumn{7}{|l|}{ Comorbidity } \\
\hline CVA & 1.71 & 1.60 & 1.82 & 1.16 & 1.08 & 1.24 \\
\hline COPD & 2.08 & 1.95 & 2.21 & 1.45 & 1.35 & 1.55 \\
\hline HTN & 2.20 & 2.05 & 2.36 & 1.42 & 1.31 & 1.53 \\
\hline $\mathrm{CHF}$ & 2.16 & 2.01 & 2.33 & 1.41 & 1.31 & 1.53 \\
\hline Diarrhea & 1.61 & 0.85 & 2.82 & 1.82 & 0.95 & 3.23 \\
\hline \multicolumn{7}{|l|}{ Medications } \\
\hline Thiazide & 1.60 & 1.34 & 1.90 & 1.16 & 0.97 & 1.38 \\
\hline Sulfonamides & 1.74 & 1.52 & 1.98 & 1.14 & 0.99 & 1.31 \\
\hline Xanthines & 2.29 & 2.02 & 2.59 & 1.42 & 1.25 & 1.62 \\
\hline Laxatives & 1.80 & 1.65 & 1.97 & 1.29 & 1.17 & 1.41 \\
\hline
\end{tabular}

UTI urinary tract infection, CVA cerebral vascular accident, COPD chronic obstructive pulmonary disease, DM diabetes mellitus, $C H F$ congestive heart failure by multiple factors including renal $[9,15,16]$, medications [17], gastrointestinal loss. Medications including thiazides, sulfonamides, xanthines, and laxatives were independently associated with hypokalemia. We hypothesized that hypokalemia developed because of renal potassium loss. The renal tubule potassium resorption was impaired after tubule injury in UTI and therefore the urine potassium loss is increased $[18,19]$. We measured the urine potassium of several UTI patients (data not shown) and the urine potassium secretion was increased in patients with UTI. More studies are needed to verify our findings.

Because hypokalemia may be related to multiple comorbidities, we include comorbidities that may be associated with both UTI $[20,21]$ and hypokalemia [22] in the analysis. DM, CKD, potassium-sparing diuretics, beta-2adrenoreceptor agonists, and steroids were associated with hypokalemia in univariable regression but not in multivariable regression. This suggests that these may be not major contributors to hypokalemia in UTI patients. A few patients $(0.1 \%)$ had diarrhea, malnutrition, or poor intake in our study. We suspected these conditions were underdiagnosed because physicians rarely coded these conditions in the diagnosis. Although the numbers of patients are limited, diarrhea was associated with hypokalemia in univariable and multivariable regressions. Duration of hospitalization, alkalosis, malnutrition, poor intake, magnesium disorder, and meropenem [23] were analyzed and was not linked to hypokalemia.

\section{Limitations}

The limitations to the study include: First, LHID only recorded the ICD-9-CM code on discharge, it is not possible to discriminate community acquired hypokalemia and hypokalemia developed in hospital. There may be many factors which may cause hypokalemia in hospital. The causal relationship can not be determined. Some of the recurrent UTI may be caused by the hypokalemia that results in the elaboration of alkaline urine, bladder dysfunction, and urinary stasis [24]. Second, the serum potassium readings and symptoms associated with hypokalemia were not available in the LHID, we were not able to report the severity of hypokalemia in this study, and symptoms associated with hypokalemia. In our clinical practice, most of the hypokalemia was asymptomatic and potassium replacement was rarely acquired. Third, we hypothesized that the renal parenchyma injury in UTI may be responsible for the development of hypokalemia. Acute pyelonephritis may be associated with more severe renal parenchyma injury than lower UTI and is more likely to develop hypokalemia. However, we did not find a significant association between acute pyelonephritis and hypokalemia. We suspected that most acute pyelonephritis may be recorded as UTI in the LHID. 


\section{Conclusion}

Urinary tract infection is associated with an increased risk of hypokalemia and measurements of serum potassium should be considered in clinical practice. UTI and hypokalemia are associated with multiple chronic diseases and medications. The association of hypokalemia and UTI is independent of comorbidities and medications.

\section{Abbreviations}

LHID: Longitudinal Health Insurance Database; ICD-9-CM: International Classification of Disease, Ninth. Revision, Clinical Modification; UTI: Urinary tract infection; CVA: Cerebral vascular accident; COPD: Chronic obstructive pulmonary disease; DM: Diabetes mellitus; CHF: Congestive heart failure; CKD: Chronic kidney disease

\section{Acknowledgements}

None.

\section{Authors' contributions}

$\mathrm{HL}$ and $\mathrm{HC}$ analyzed and interpreted the data. YF and $\mathrm{CY} 1$ made substantia contributions to the conception. CY2 have drafted the work. AL substantively revised the work. CY1 corresponds to Chien-Yeh Hsu and CY2 corresponds to Che-Yi Chou. All authors read and approved the final manuscript.

\section{Funding}

None.

\section{Availability of data and materials}

The datasets used and/or analyzed during the current study are available from the corresponding author on reasonable request.

\section{Ethics approval and consent to participate}

The dataset is a de-identified secondary data and is released to the public for research purposes. A review of the institutional review board is not required in conducting this study.

\section{Consent for publication}

Not applicable.

\section{Competing interests}

The authors declare that they have no competing interests.

\section{Author details}

'Department of Neurology, Sijhih Cathay General Hospital, No.2, Ln. 59 , Jiancheng Rd., Sijhih Dist, New Taipei City 221, Taiwan. ${ }^{2}$ Graduate Institute of Biomedical Informatics, Taipei Medical University, No. 252, Wu-Xing Street, Taipei 110, Taiwan. ${ }^{3}$ Department of Pediatrics, School of Medicine, College of Medicine, Taipei Medical University, No. 252, Wu-Xing Street, Taipei 110, Taiwan. ${ }^{4}$ Department of Laboratory Medicine, Taipei Medical University Hospital, No. 252, Wu-Xing Street, Taipei 110, Taiwan. ${ }^{5}$ Department of Information Management, National Taipei University of Nursing and Health Science, No. 365, Mingde Rd, Taipei 112, Taiwan. ${ }^{6}$ Division of Nephrology, Asia University Hospital, NO 222, Fuxin Rd, Wufeng Dist, Taichung 413, Taiwan. ${ }^{7}$ Department of Post-baccalaureate Veterinary Medicine, Asia University, NO 222, Fuxin Rd, Wufeng Dist, Taichung 413, Taiwan. ${ }^{8}$ Division of Nephrology, China Medical University Hospital, Tacihung, Taiwan.

Received: 15 February 2020 Accepted: 14 July 2020

Published online: 20 July 2020

\section{References}

1. Skogestad J, Aronsen JM. Hypokalemia-induced arrhythmias and heart failure: new insights and implications for therapy. Front Physiol. 2018;9:1500.

2. Jensen HK, Brabrand M, Vinholt PJ, Hallas J, Lassen AT. Hypokalemia in acute medical patients: risk factors and prognosis. Am J Med. 2015;128(1):60-7 e61.

3. Collins AJ, Pitt B, Reaven N, Funk S, McGaughey K, Wilson D, et al. Association of Serum Potassium with all-cause mortality in patients with and without heart failure, chronic kidney disease, and/or diabetes. Am J Nephrol. 2017:46(3):213-21.

4. Kolman KB. Cystitis and pyelonephritis: diagnosis, treatment, and prevention Prim Care. 2019:46(2):191-202.

5. Foxman B. Urinary tract infection syndromes: occurrence, recurrence, bacteriology, risk factors, and disease burden. Infect Dis Clin N Am. 2014; 28(1):1-13.

6. Moskowitz D, Lee U. Patient distress in women with recurrent urinary tract infections: how can physicians better meet patients needs? Curr Urol Rep. 2018;19(12):97.

7. Wu KL, Cheng CJ, Sung CC, Tseng MH, Hsu YJ, Yang SS, et al. Identification of the causes for chronic hypokalemia: importance of urinary sodium and chloride excretion. Am J Med. 2017:130(7):846-55.

8. Melnick JZ, Preisig PA, Haynes S, Pak CY, Sakhaee K, Alpern RJ. Converting enzyme inhibition causes hypocitraturia independent of acidosis or hypokalemia. Kidney Int. 1998;54(5):1670-4.

9. Weiner ID, Wingo CS. Hypokalemia--consequences, causes, and correction. J Am Soc Nephrol. 1997:8(7):1179-88.

10. Lindeman RD. Hypokalemia: causes, consequences and correction. Am J Med Sci. 1976:272(1):5-17.

11. Kogika MM, de Morais HA. A quick reference on hypokalemia. Vet Clin North Am Small Anim Pract. 2017:47(2):229-34.

12. Shen AL, Lin HL, Lin HC, Tseng YF, Hsu CY, Chou CY. Increased risk of bullous pemphigoid after first-ever stroke: a population-based study. Neurodegener Dis. 2017;17(4-5):166-70.

13. Foxman B. Recurring urinary tract infection: incidence and risk factors. Am J Public Health. 1990;80(3):331-3.

14. Zhou X, Zhang Z, Li J, Ye Y, Tang G, Ding B. Cardiac arrest among patients with infections. Clin Microbiol Infect. 2017;23(10):782

15. Stein JH. Hypokalemia. Common and uncommon causes. Hosp Pract (Off Ed). 1988:23(3A):55-64 66, 70

16. Steigerwalt SP. Unraveling the causes of hypertension and hypokalemia. Hosp Pract (1995). 1995:30(7):67-71 74-65, 79.

17. Buckley MS, Leblanc JM, Cawley MJ. Electrolyte disturbances associated with commonly prescribed medications in the intensive care unit. Crit Care Med. 2010;38(6 Suppl):S253-64.

18. Melis K, Vandevivere J, Hoskens C, Vervaet A, Sand A, Van Acker KJ. Involvement of the renal parenchyma in acute urinary tract infection: the contribution of 99mTc dimercaptosuccinic acid scan. Eur J Pediatr. 1992 151(7):536-9.

19. Ronald A. The etiology of urinary tract infection: traditional and emerging pathogens. Am J Med. 2002;113(Suppl 1A):14S-9S.

20. Medina M, Castillo-Pino E. An introduction to the epidemiology and burden of urinary tract infections. Ther Adv Urol. 2019:11:1756287219832172.

21. Mody L, Juthani-Mehta M. Urinary tract infections in older women: a clinical review. JAMA. 2014;311(8):844-54.

22. Marti G, Schwarz C, Leichtle AB, Fiedler GM, Arampatzis S, Exadaktylos AK, et al. Etiology and symptoms of severe hypokalemia in emergency department patients. Eur J Emerg Med. 2014;21(1):46-51.

23. Zaki SA, Shanbag P. Meropenem-induced hypokalemia and metabolic alkalosis. Indian J Pharm. 2012:44(2):276-7.

24. Cohen PG. The pathogenesis of recurrent urinary tract infection: the bowel, bladder hypokalemia connection. Med Hypotheses. 1983;10(1):1-4.

\section{Publisher's Note}

Springer Nature remains neutral with regard to jurisdictional claims in published maps and institutional affiliations.

Ready to submit your research? Choose BMC and benefit from:

- fast, convenient online submission

- thorough peer review by experienced researchers in your field

- rapid publication on acceptance

- support for research data, including large and complex data types

- gold Open Access which fosters wider collaboration and increased citations

- maximum visibility for your research: over $100 \mathrm{M}$ website views per year

At $\mathrm{BMC}$, research is always in progress.

Learn more biomedcentral.com/submissions 